PROCEEDINGS OF THE

AMERICAN MATHEMATICAL SOCIETY

Volume 136, Number 7, July 2008, Pages 2439-2443

S 0002-9939(08)09472-0

Article electronically published on March 6, 2008

\title{
REGULARITY FOR THE NAVIER-STOKES EQUATIONS WITH SLIP BOUNDARY CONDITION
}

\author{
HYEONG-OHK BAE AND BUM JA JIN
}

(Communicated by David S. Tartakoff)

\begin{abstract}
For the Navier-Stokes equations with slip boundary conditions, we obtain the pressure in terms of the velocity. Based on the representation, we consider the relationship in the sense of regularity between the Navier-Stokes equations in the whole space and those in the half space with slip boundary data.
\end{abstract}

\section{INTRODUCTION}

In this paper we consider Navier-Stokes equations with slip boundary condition. Consider the Navier-Stokes equations in $\mathbb{R}_{+}^{3} \times(0, T)$ :

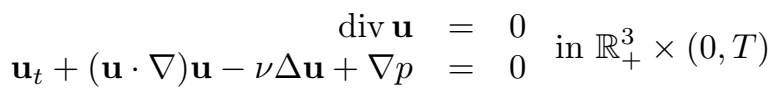

for some positive $T>0$ satisfying the initial boundary condition;

$$
\partial_{3} u_{1}=\partial_{3} u_{2}=u_{3}=0 \text { on } x_{3}=0, \quad \mathbf{u}(\mathbf{x}) \rightarrow \mathbf{0} \text { as }|\mathbf{x}| \rightarrow \infty,
$$

and $\mathbf{u}(\mathbf{x}, 0)=\mathbf{u}_{0}(\mathbf{x})=\left(u_{0,1}(\mathbf{x}), u_{0,2}(\mathbf{x}), u_{0,3}(\mathbf{x})\right)$, where $\mathbf{u}_{0} \in L^{2}\left(\mathbb{R}_{+}^{3}\right)$ with div $\mathbf{u}_{0}=$ 0 in $\mathbb{R}_{+}^{3}$. The existence of the suitable weak solution $\mathbf{u}$ in $L^{2}\left(0, T ; H_{0}^{1}\left(\mathbb{R}_{+}^{3}\right)\right)$ was mentioned in [7] for the domain $\mathbb{R}^{3}$ or a bounded domain, in [20] and [1] for the half space, and [11] for the exterior domain. The suitably weak solution $(\mathbf{u}, p)$ satisfies Navier-Stokes equations in the sense of distribution, with the properties of global energy inequality

$$
\|\mathbf{u}(\cdot, t)\|_{L^{2}\left(\mathbb{R}_{+}^{3}\right)}^{2}+\int_{0}^{t}\|\nabla \mathbf{u}\|_{L^{2}\left(\mathbb{R}_{+}^{3}\right.}^{2} \leq\left\|\mathbf{u}_{0}\right\|_{L^{2}\left(\mathbb{R}_{+}^{3}\right)}^{2}
$$

and localized energy inequality:

$$
2 \iint|\nabla \mathbf{u}|^{2} \phi \leq \int\left[|u|^{2}\left(\phi_{t}+\Delta \phi\right)+\left(|\mathbf{u}|^{2}+2 p\right) \mathbf{u} \cdot \nabla \phi\right]
$$

for each real-valued $\phi \in C_{0}^{\infty}\left(\mathbb{R}_{+}^{3} \times(0, T)\right)$ with $\phi \geq 0$.

The slip boundary condition has been considered in terms of the study of the free boundary problem by several mathematicians (see [24, [25, [12, [17, [13, [19],

Received by the editors January 14, 2006, and, in revised form, September 17, 2006.

2000 Mathematics Subject Classification. Primary 35Q30, 76D07.

Key words and phrases. Navier-Stokes, pressure representation, slip boundary condition, regularity.

The first author was supported by grant (R05-2002-000-00002-0(2002)) from the Basic Research Program of the Korea Science \& Engineering Foundation.

(C)2008 American Mathematical Society Reverts to public domain 28 years from publication 
23], etc.), and recently Beirao da Veiga [2, 3, 4, 5] has considered its regularity. In particular, in [4] he derived the regularity result for the weak solutions of (1.1) and (1.2), which is an extension of the regularity result in $\mathbb{R}^{3}$ by P. Constantin and C. Fefferman [10]. (See also [6].)

In this work, we represent the pressure by the velocity as a first step in the half space $\mathbb{R}_{+}^{3}$. Moreover, we show that the extension of $(\mathbf{u}, p)$ by (even, even, odd, even) reflection will give a solution of the Navier-Stokes equation in the whole space. Then we will conclude that almost every regularity result obtained for the solution of the Navier-Stokes equations in the whole space hold for the solution of the Navier-Stokes equations with slip boundary conditions in the half space. We emphasize that our observation will give another, but simpler, reasoning for the regularity result in [4].

\section{MAin Results}

Proposition 2.1. Suppose $\mathbf{u}=\left(u_{1}, u_{2}, u_{3}\right), p$ is a solution of the incompressible Navier-Stokes equation (1.1) with slip boundary condition (1.2) in the half space $\mathbb{R}_{+}^{3}$ with initial data $\mathbf{u}_{0}=\left(u_{0,1}, u_{0,2}, u_{0,3}\right)$, where $\nabla \cdot \mathbf{u}_{0}=0$ in $\mathbb{R}_{+}^{3}, \partial_{3} u_{0,1}=\partial_{3} u_{0,2}=$ $u_{0,3}=0$ on $x_{3}=0$, and $\lim _{|\mathbf{x}| \rightarrow \infty} \mathbf{u}_{0}=0$. Then $p$ has the following representation. For almost all time $t \in(0, T)$

$$
p(\mathbf{x}, t)=\frac{-\delta_{i j}}{3}\left(u_{i}^{*} u_{j}^{*}\right)(\mathbf{x}, t)+\frac{3}{4 \pi} \int_{\mathbb{R}^{3}} \frac{\partial^{2}}{\partial y_{i} \partial y_{j}} \frac{1}{|\mathbf{x}-\mathbf{y}|}\left[\left(u_{i}^{*} u_{j}^{*}\right)(\mathbf{y}, t)\right] d \mathbf{y}
$$

in the sense of distributions, where $\delta_{i j}$ is the Kronecker delta function. Here, $\mathbf{u}^{*}(\mathbf{y})=\mathbf{u}(\mathbf{y})$ for $y_{3}>0, u_{1}^{*}(\mathbf{y}, t)=u_{1}\left(\mathbf{y}^{*}, t\right), u_{2}^{*}(\mathbf{y}, t)=u_{2}\left(\mathbf{y}^{*}, t\right), u_{3}^{*}(\mathbf{y}, t)=$ $-u_{3}\left(\mathbf{y}^{*}, t\right)$ for $y_{3}<0$, and $\mathbf{y}^{*}=\left(y_{1}, y_{2},-y_{3}\right)$.

Proof. Let $Q=\mathbb{R}_{+}^{3} \cup\left\{x_{3}=0\right\}, Q_{T}=Q \times[0, T)$. Without loss of generality, assume $\mathbf{u}_{0} \in C^{1}(Q)$ and $\mathbf{u} \in L^{2}\left(0, T ; C^{1}(Q)\right)$ considering the existence space $L^{2}\left(0, T ; H^{1}(Q)\right)$.

Set $\mathbf{f}(\mathbf{x}, t)=-[(\mathbf{u} \cdot \nabla) \mathbf{u}](\mathbf{x}, t)$ for $x_{3} \geq 0$. Define $\mathbf{f}^{*}=\left(f_{1}^{*}, f_{2}^{*}, f_{3}^{*}\right)$ by

$$
\begin{aligned}
& f_{1}^{*}(\mathbf{x}, t)=\left\{\begin{aligned}
f_{1}(\mathbf{x}, t), & \text { if } x_{3} \geq 0, \\
f_{1}\left(\mathbf{x}^{*}, t\right), & \text { if } x_{3}<0,
\end{aligned}\right. \\
& f_{2}^{*}(\mathbf{x}, t)=\left\{\begin{aligned}
f_{2}(\mathbf{x}, t), & \text { if } x_{3} \geq 0, \\
f_{2}\left(\mathbf{x}^{*}, t\right), & \text { if } x_{3}<0,
\end{aligned}\right. \\
& f_{3}^{*}(\mathbf{x}, t)=\left\{\begin{aligned}
f_{3}(\mathbf{x}, t), & \text { if } x_{3} \geq 0, \\
-f_{3}\left(\mathbf{x}^{*}, t\right), & \text { if } x_{3}<0 .
\end{aligned}\right.
\end{aligned}
$$

Here $\mathbf{x}^{*}$ is the even-even-odd extension of $\mathbf{x}$ like $\mathbf{y}^{*}$. Since $u_{3}=0$ on $x_{3}=0$, one has $\partial_{1} u_{3}=\partial_{2} u_{3}=0$ on $x_{3}=0$, therefore $f_{3}=0$. By noting $\partial_{3} u_{0,1}=\partial_{3} u_{0,2}=u_{0,3}=0$, and $f_{3}=0$ on $x_{3}=0$, it follows that $\mathbf{u}_{0}^{*} \in C\left(\mathbb{R}^{3}\right)$ and $\mathbf{f}^{*} \in C\left(\mathbb{R}^{3}\right)$.

Observe that $f_{1}^{*}(\mathbf{x}, t)=-\left[\left(\mathbf{u}^{*} \cdot \nabla\right) u_{1}^{*}\right](\mathbf{x}, t), f_{2}^{*}(\mathbf{x}, t)=-\left[\left(\mathbf{u}^{*} \cdot \nabla\right) u_{2}^{*}\right](\mathbf{x}, t)$, and $f_{3}^{*}(\mathbf{x}, t)=-\left[\left(\mathbf{u}^{*} \cdot \nabla\right) u_{3}^{*}\right](\mathbf{x}, t)$ for $x_{3}<0$. Hence, $\mathbf{f}^{*}(\mathbf{x}, t)=-\operatorname{div}(\mathbf{u} \otimes \mathbf{u})(\mathbf{x}, t)$ for $x_{3}>0$ and $\mathbf{f}^{*}(\mathbf{x}, t)=-\operatorname{div}\left(\mathbf{u}^{*} \otimes \mathbf{u}^{*}\right)(\mathbf{x}, t)$ for all $x_{3}<0$. Since $\partial_{3} u_{1}=\partial_{3} u_{2}=u_{3}=$ 0 on $x_{3}=0$, it follows that

$$
\mathbf{f}^{*}(\mathbf{x}, t)=-\operatorname{div}\left(\mathbf{u}^{*} \otimes \mathbf{u}^{*}\right)(\mathbf{x}, t)
$$

in the sense of distributions. 
Now we construct $(\mathbf{v}, q)$ as a solution of the Stokes system in $\mathbb{R}^{3}$,



with initial data $\mathbf{v}(\mathbf{x}, 0)=\mathbf{u}_{0}^{*}(\mathbf{x})$ and infinity condition $\mathbf{v}(\mathbf{x}, t) \rightarrow \mathbf{0}$ as $|\mathbf{x}| \rightarrow \infty$.

Then $q$ satisfies the Laplace equation $\Delta q(\mathbf{x}, t)=\operatorname{div} \mathbf{f}^{*}(\mathbf{x}, t)$ in $\mathbb{R}^{3} \times(0, T)$. We try to find $q$ integrable. By integral representation, $q$ is expressed by

$$
\begin{aligned}
q(\mathbf{x}, t) & =-\frac{3}{4 \pi} \int_{\mathbb{R}^{3}} \frac{1}{|\mathbf{x}-\mathbf{y}|} \partial_{j} f_{j}^{*}(\mathbf{y}, t) d \mathbf{y} \\
& =\frac{-\delta_{i j}}{3}\left(u_{i}^{*} u_{j}^{*}\right)(\mathbf{x}, t)+\frac{3}{4 \pi} \int_{\mathbb{R}^{3}} \partial_{i} \partial_{j} \frac{1}{|\mathbf{x}-\mathbf{y}|}\left(u_{i}^{*} u_{j}^{*}\right)(\mathbf{y}, t) d \mathbf{y},
\end{aligned}
$$

where $\delta_{i j}$ is the Kronecker delta function.

Our aim is to show $\mathbf{u} \equiv \mathbf{v}$ and $p \equiv q+c_{0}$ in the half space $\mathbb{R}_{+}^{3} \times(0, T)$. Set $W_{1}(\mathbf{x}, t)=v_{1}(\mathbf{x}, t)-v_{1}\left(\mathbf{x}^{*}, t\right), W_{2}(\mathbf{x}, t)=v_{2}(\mathbf{x}, t)-v_{2}\left(\mathbf{x}^{*}, t\right), W_{3}(\mathbf{x}, t)=v_{3}(\mathbf{x}, t)+$ $v_{3}\left(\mathbf{x}^{*}, t\right)$ and $Q(\mathbf{x}, t)=q(\mathbf{x}, t)-q\left(\mathbf{x}^{*}, t\right)$.

Then $\mathbf{W}$ and $Q$ satisfy

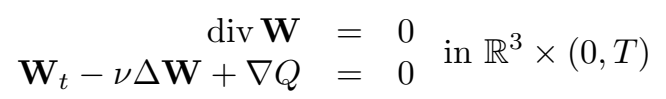

and $\mathbf{W}(\mathbf{x}, 0)=\mathbf{0}, W(\mathbf{x}, t) \rightarrow \mathbf{0}$ as $|\mathbf{x}| \rightarrow 0$. By the uniqueness of the Stokes solution, we conclude that $\mathbf{W} \equiv 0$. Therefore, $v_{1}(\mathbf{x}, t)=v_{1}\left(\mathbf{x}^{*}, t\right), v_{2}(\mathbf{x}, t)=v_{2}\left(\mathbf{x}^{*}, t\right)$ and $v_{3}(\mathbf{x}, t)=-v_{3}\left(\mathbf{x}^{*}, t\right)$. From the above identity, we deduce that $\left(\partial_{3} v_{1}\right)\left(x_{1}, x_{2}, 0, t\right)=$ $\left(\partial_{3} v_{2}\right)\left(x_{1}, x_{2}, 0, t\right)=v_{3}\left(x_{1}, x_{2}, 0, t\right)=0$.

Now, it only remains to show $\mathbf{v} \equiv \mathbf{u}$ and $p=q+c_{0}$ for $x_{3}>0$. Let $\mathbf{U}=$ $\mathbf{u}-\mathbf{v}, P=p-q$. Then $\mathbf{U}(\mathbf{x}, 0)=\mathbf{0},\left(\partial_{3} U_{1}\right)\left(x_{1}, x_{2}, 0, t\right)=\left(\partial_{3} U_{2}\right)\left(x_{1}, x_{2}, 0, t\right)=$ $U_{3}\left(x_{1}, x_{2}, 0, t\right)=0$ and $\mathbf{U}(\mathbf{x}, t) \rightarrow 0$ as $|\mathbf{x}| \rightarrow 0$. Moreover, $\mathbf{U}$ and $P$ satisfy

$$
\begin{array}{r}
\operatorname{div} \mathbf{U}=0 \\
\mathbf{U}_{t}-\nu \Delta \mathbf{U}+\nabla P=0
\end{array} \text { in } \mathbb{R}_{+}^{3} \times(0, T) .
$$

From the uniqueness of the Stokes solution, we again conclude that $\mathbf{U} \equiv 0$ in $x_{3}>0$. This again implies that $P \equiv$ const and that $p=q+c_{0}$ for a constant $c_{0}$. Since we try to find $q$ integrable, we may ignore the constant $c_{0}$.

Remark. If $p \in L^{r}\left(\mathbb{R}_{+}^{3}\right)$, then the constant $c_{0}$ is zero, so that we have $\|p\|_{L^{r}} \leq$ $c\|\mathbf{u}\|_{L^{2 r}}^{2}$ for $1<r<\infty$ for a constant $c$ depending only on $r$.

During the proof of Proposition 2.1. we also observe that $\mathbf{u}^{*}$ and $p^{*}$ satisfy the Navier-Stokes equations in the whole space with initial velocity $\mathbf{u}_{0}^{*}$, and we state it as a corollary.

Corollary 2.2. Expand $\mathbf{u}, p$ to the whole space by

$$
\left(\mathbf{u}^{*}, p^{*}\right)(\mathbf{y}, t)= \begin{cases}(\mathbf{u}, p)(\mathbf{y}, t) & \text { for } y_{3}>0, \\ \left(u_{1}, u_{2},-u_{3}, p\right)\left(\mathbf{y}^{*}, t\right) & \text { for } y_{3}<0,\end{cases}
$$

where $\mathbf{y}^{*}=\left(y_{1}, y_{2},-y_{3}\right)$. Then $\mathbf{u}^{*}$ and $p^{*}$ satisfy the Navier-Stokes equations in the whole space with initial velocity $\mathbf{u}_{0}^{*}$, where

$$
\mathbf{u}_{0}^{*}(\mathbf{y}, t)= \begin{cases}\mathbf{u}_{0}(\mathbf{y}) & \text { for } y_{3}>0 \\ \left(u_{0,1}, u_{0,2},-u_{0,3}\right)\left(\mathbf{y}^{*}\right) & \text { for } y_{3}<0\end{cases}
$$

Therefore, most regularity results for the Navier-Stokes equations in the whole space are also applicable to those in the half space as follows. 
Theorem 2.3. Suppose $(\mathbf{u}, p)$ is a suitably weak solution of the Navier-Stokes equations (1.1) in the half space with slip boundary (1.2). There is $\epsilon_{0}>0$, and $C_{k}$ such that

$$
\int_{Q_{1}^{+}}|\mathbf{u}|^{3}+|p|^{3 / 2} d x d t<\epsilon_{0}
$$

implies that

$$
\sup _{Q_{1 / 2}^{+}}\left|\nabla^{k} \mathbf{u}\right| \leq C_{k} .
$$

Proof. Let us recall that $\mathbf{u}^{*}, p^{*}$ defined in Corollary 2.2 satisfies Navier-Stokes equations in the whole space. Hence by Proposition 1 in [7] we have that there is $\epsilon_{1}$ and $C_{k}$ such that

$$
\int_{Q_{1}}\left|\mathbf{u}^{*}\right|^{3}+\left|p^{*}\right|^{3 / 2} d x d t<\epsilon_{1}
$$

implies

$$
\sup _{Q_{1 / 2}}\left|\nabla^{k} \mathbf{u}^{*}\right| \leq C_{k}
$$

Observe that

$$
\int_{Q_{1}}\left|\mathbf{u}^{*}\right|^{3}+\left|p^{*}\right|^{3 / 2} d x d t=2 \int_{Q_{1}^{+}}|\mathbf{u}|^{3}+|p|^{3 / 2} d x d t .
$$

Hence if we take $\epsilon_{0}=\frac{1}{2} \epsilon_{1}$, then we complete the proof. (See also [15] and [20.)

Remark. By the same argument as in Theorem 2.3, the following results also hold for the solutions of the Navier-Stokes equations (1.1) in the half space with boundary condition (1.2):

1. The only self-similar solution of the Navier-Stokes equation (1.1) with slip boundary condition (1.2) is equal to zero. (See [18] and [26].)

2. If $\mathbf{u} \in L^{q, s}\left(Q_{1}^{+}\right), \frac{3}{q}+\frac{2}{s} \leq 1, q \geq 3$, then $\mathbf{u}$ is smooth in $Q_{1 / 2}^{+}$. (See [1], [9], [14, 20] and [21.)

3. Let $\omega=\nabla \times \mathbf{u}$. Let us denote by $\theta(x, y, t)$ the angle between the vorticity $\omega$ at two distinct points $x$ and $y$ at time $t$. Assume that $|\sin \theta(x, y, t)| \leq$ $c|x-y|^{\beta}, \beta \in[0,1 / 2]$, in the region where the vorticity at both points $x$ and $y$ is larger than an arbitrary fixed positive constant $K$. Moreover, suppose that $\omega \in L^{2}\left(0, T ; L^{r}\right)$, where $r=\frac{3}{\beta+t}$. Then $\mathbf{u}$ is regular. (See [10, [6] and [8].)

\section{REFERENCES}

[1] H.-O. Bae, H.J. Choe, and B.J. Jin, Pressure Representation and Boundary Regularity of the Navier-Stokes Equations with Slip Boundary Condition, to appear in J. Diff. Equations.

[2] H. Beirão da Veiga, Regularity for Stokes and generalized Stokes systems under nonhomogeneous slip-type boundary conditions, Adv. Differential Equations 9 (2004), no. 9-10, 1079 1114. MR2098066 (2006f:35213)

[3] H. Beirão da Veiga, On the regularity of flows with Ladyzhenskaya shear-dependent viscosity and slip or nonslip boundary conditions, Comm. Pure Appl. Math. 58 (2005), no. 4, 552-577. MR.2119869 (2005k:35329)

[4] H. Beirão da Veiga, Vorticity and regularity for flows under the Navier boundary condition, Comm. Pure Appl. Analysis 5 (2006), no. 4, 907-918. MR2246015 (2007h:35248)

[5] H. Beirão da Veiga, Regularity of solutions to a non-homogeneous boundary value problem for general Stokes systems in $R_{+}^{n}$, Math. Ann. 331 (2005), 203-217. MR2107444(2006a:35230) 
[6] H. Beirão da Veiga and L.C. Berselli, On the regularizing effect of the vorticity direction in incompressible viscous flows, Differ. Integral Equ. 15 (2002), 345-356. MR.1870646 (2002k:35248)

[7] L. Caffarelli, J. Kohn and L. Nirenberg, Partial regularity for suitable weak solutions of the Navier-Stokes equations, Comm. Pure Appl. Math. 35 (1982), 771-831. MR673830 (84m:35097)

[8] D. Chae and H.J. Choe, Regularity of solutions to the Navier-Stokes equation, Electronic J. Diff. Eqns. 5 (1999), no. 1, 1-7. MR1673067 (99m:35184)

[9] H.J. Choe, Boundary regularity of weak solutions of the Navier-Stokes equations, J. Diff. Eqns. 149 (1998), no. 2, 211-247. MR.1646239(99j:35161)

[10] P. Constantin and C. Fefferman, Direction of vorticity and the problem of global regularity for the Navier-Stokes equations, Indiana Univ. Math. J. 42 (1993), no. 3, 775-789. MR1254117 (95j:35169)

[11] G.P. Galdi and P. Maremonti, Monotonic decreasing and asymptotic behavior of the kinetic energy for weak solutions of the Navier-Stokes equations in exterior domain, Arch. Ratl. Mech. Anal. 94 (1986), 253-266. MR846064 (87j:35295)

[12] S. Itoh and A. Tani, The initial value problem for the non-homogeneous Navier-Stokes equations with general slip boundary condition, Proc. Roy. Soc. Edinburgh Sect. A 130 (2000), no. 4, 827-835. MR1776680 (2001g:76016)

[13] V. John, Slip with friction and penetration with resistence boundary conditions for the Navier-Stokes equations-numerical tests and aspects of the implementation, J. Comp. Appl. Math. 147 (2002), 287-300. MR1933597(2003h:76033)

[14] K. Kang, On boundary regularity for the Stokes and Navier-Stokes equations, thesis, University of Minnesota (2002).

[15] F. Lin, A new proof of the Caffarelli-Kohn-Nirenberg theorem, Comm. Pure Appl. Math. 51 (1998), no. 3, 241-257. MR1488514 (98k:35151)

[16] P. Maremonti, Some theorems of existence for solutions of the Navier-Stokes equations with slip boundary conditions in half-space, Ricerche di Matematica, vol. XL, no. 1 (1991), 81-135. MR1191888 (94b:35214)

[17] V.G. Mazja, B.A. Plamenevskii and L.T. Stupyalis, The three-dimensional problem of steady state motion of a fluid with a free surface, Trans. Am. Math. Soc. 123 (1984), 171-268. MR0548252 (81m:76019)

[18] J. Nečas, M. Rǔžiča, V. Sverák, On Leray's self-similar solutions of the Navier-Stokes equations, Acta Math. 176 (1996), 283-294. MR1397564 (97f:35165)

[19] H. Saito and L.E. Scriven, Study of the coating flow by the finite element method, J. Comput. Phys. 42 (1981), 53-76.

[20] G.A. Seregin, Local regularity of suitable weak solutions to the Navier-Stokes equations near the boundary, J. Math. Fluid Mech. 4 (2002), no. 1, 1-29. MR1891072 (2003a:35152)

[21] G.A. Seregin and V. Šverak, On smoothness of suitable weak solutions to the Navier-Stokes equations, Zap. Nauchn. Sem. S.-Peterburg. Otdel. Mat. Inst. Steklov (POMI) 306 (2003). MR2065503 (2005f:35249)

[22] J. Serrin, On the interior regularity of weak solutions of the Navier-Stokes equations, Arch. Ration. Mech. Anal. 9 (1962), 187-195. MR0136885(25:346)

[23] J. Silliman and L.E. Scriven, Separating flow near a static contact line: slip at a wall and shape of a free surface, J. Comput. Phys. 34 (1980), 287-313. MR562365 (81b:76026)

[24] V.A. Solonnikov, Solvability of three dimensional problems with free boundary for a stationary system of Navier-Stokes equations, J. Sov. Math. 21 (1983), 427-450.

[25] V.A. Solonnikov and V.E. Scadilov, A certain boundary value problem for the stationary system of Navier-Stokes equations, Trudy Mat. Inst. Steklov 125 (1973), 196-210, 235. MR0364910 (51:1164)

[26] T. Tsai, On Leray self-similar solutions of the Navier-Stokes equations satisfying local energy estimates, Arch. Rational Mech. Anal. 143 (1998), 29-51. MR.1643650 (99j:35171)

Department of Mathematics, Ajou University, Suwon 443-749, Korea

E-mail address: hobae@ajou.ac.kr

Department of Mathematics, Mokpo National University, Muan 534-729, Korea

E-mail address: bumjajin@hanmail.net 